\title{
Relaciones entre estrés psicosocial y lesiones deportivas en tenistas
}

\section{Psychosocial stress and sport injuries in tennis players}

Recibido: junio 3 de 2009 ｜ Revisado: septiembre 6 de 2009 | Aceptado: febrero 14 de 2010

\author{
Aurelio Olmedilla Zafra* \\ Universidad de Murcia, España \\ Joel M. PRIETO ANDREU** \\ Universidad Católica San Antonio de Murcia, España \\ AMADOR Blas REDONDO \\ Sociedad Murciana de Psicología de la Actividad \\ Física y el Deporte
}

\section{RESUMEN}

El propósito de este trabajo fue evaluar el estrés social percibido y su relación con las lesiones deportivas, en jugadores de tenis. Sesenta y tres tenistas masculinos, con una media de edad de 31.62 años $(D T=8.93)$, pertenecientes a Clubes Deportivos de la Provincia de Alicante (España), cumplimentaron instrumentos de estrés psicosocial y de lesiones sufridas durante el año anterior a la evaluación. Los resultados indican que los sucesos vitales experimentados, se relacionan con algunas de las lesiones sufridas por los tenistas. Se han encontrado relaciones entre el grado de tensión psicológica experimentada y la evaluación negativa de esta tensión, con el tipo (lesiones en muñecas, tobillos y esguinces), y la gravedad de las lesiones (lesiones leves).

Palabras clave autores

Estrés psicosocial, lesiones, tenistas, diseño descriptivo correlacional, deporte.

Palabras clave descriptors

Pruebas psicológicas, psicología del deporte, investigación cuantitativa.

\section{A B S T R A C T}

The purpose of this study was to assess the perceived social stress and its relation to sports injuries in tennis players. Sixty-three male tennis players, with a mean age of 31.62 years $(S D=8.93)$, Sports Clubs belonging to the Province of Alicante (Spain) completed instruments of psychosocial stress and injuries sustained during the previous year the assessment. The results indicate that life events experienced was related to some of the injuries suffered by players. Relationships were found between the degree of psychological stress experienced and the negative evaluation of this strain, the type (lesions on wrists, ankles and sprains), and severity of injuries (minor injuries). Key words authors

Psychosocial stress, sports injuries, tennis players, correlational descriptive design, sport.

Key words plus

Psychological tests, sport psychology, quantitative research. 
Los factores psicológicos parecen desempeñar un importante papel en la aparición de lesiones deportivas. La literatura científica ha proporcionado, en los últimos años, evidencia empírica suficiente que permite comprender mejor las relaciones entre Psicología y lesión (Davis, 1991; Junge, 2000; Olmedilla, Ortín \& De la Vega, 2006; Ortín, Garcés de los Fayos \& Olmedilla, 2010; Udry \& Andersen, 2002). Uno de los aspectos clave de estudio ha sido el papel que el estrés juega en esta relación. El estrés es uno de los factores psicosociales más estudiados, sobre todo por su relación con la aparición de cierto tipo de enfermedades (Malhotra \& Mehta, 2008), el manejo y adaptación a situaciones difíciles y el desempeño en general, en una gran diversidad de poblaciones diferentes, como trabajadores (Fernández, Siegrist, Rödel \& Hernández, 2003), estudiantes (Feldman, et al., 2008) o deportistas (Díaz, Buceta \& Bueno, 2004). En este trabajo se conceptualiza el estrés desde la perspectiva fenomenológica propuesta por Lazarus y Folkman (1986), la cual subraya la importancia de la relación individuo-ambiente y la evaluación que se hace del mismo. En la actualidad no se puede dudar de que el estrés constituye un importante factor de riesgo, tanto para la salud física, como para la salud mental (Sandín, 1999). El estrés psicosocial está caracterizado, básicamente, por la asociación a estresores de tipo psicosocial, como los sucesos vitales, adversidades de diferentes tipos, etc. (Kaplan, 1996).

La mayor parte de la investigación sobre el estrés y la lesión deportiva, se ha llevado a cabo partiendo del modelo de Andersen y Williams (1988), revisado más tarde (Williams \& Andersen, 1998), modelo teórico de interacción entre las lesiones y el estrés, tomando este último como un concepto amplio, que ya fue esbozado en un trabajo anterior (Williams \& Andersen, 1986). En el análisis de la relación entre el estrés y las lesiones deportivas, los sucesos vitales han supuesto uno de los aspectos más estudiados. En general, la investigación de los sucesos vitales se ha llevado a cabo sobre todo en el ámbito anglosajón, ya que no sucede lo mismo en el ámbito latinoamericano, y habitualmente desde dos perspectivas. La que considera aquellos sucesos de gran impacto para el sujeto, los llamados sucesos vitales estresantes, que hacen referencia a la experiencia objetiva, discreta y observable que altera el funcionamiento usual del individuo, provocando en este una necesidad de ajuste. Y la que considera aquellos estresores psicosociales imbricados en la experiencia diaria de los sujetos, que hacen referencia a las experiencias cotidianas, denominados también como estrés diario o problemas diarios (Holmes, 1970). En este sentido, la clasificación de Chiriboga (1989) caracterizó conceptualmente las diferentes formas de estresores psicosociales, además de analizarlas, sugiriendo tres tipos de niveles: nivel micro, aquel que incluye estresores psicosociales imbricados en las experiencias diarias de las personas; nivel moderado, referido a situaciones que generalmente son menos frecuentes que los estresares del nivel micro, pero que también actúan directamente sobre el individuo y nivel macro, referido a estresores del sistema macrosocial.

El interés inicial en las situaciones cotidianas proviene del trabajo de Holmes y Rahe (1967), en el que descubrieron que los individuos con alto nivel de estrés, parecían, en general, ser más propensos a enfermar que aquellos con un nivel más bajo (Miller, 1988; Sarason, Johnson \& Siegel, 1978; Savery \& Wooden, 1994; Theorell, 1992). Más tarde, se encontró una relación similar para la existencia de accidentes (Selzer \& Vinokur, 1974; Stuart \& Brown, 1981). A raíz de los resultados que apoyaban la relación entre el estrés cotidiano y los accidentes, se razonó que este estrés podría influir en otra forma de enfermedad, que es la lesión deportiva. Sin embargo, respecto al estudio de los problemas diarios, los primeros trabajos que lo abordaron no encontraron relación directa con la vulnerabilidad a la lesión, quizá por cuestiones metodológicas (Blackwell \& McCullagh, 1990; Hanson, McCullagh \& Tonymon, 1992; Meyer, 1995), como por ejemplo medir una sola vez cada suceso, cuando este tipo de problemas son de naturaleza cambiante y precisarían de otro tipo de evaluación, como señalan Byrd (1993 citado en Williams \& Andersen, 1998) y Fawkner (1995); en estos dos trabajos se encontró que los deportistas eran más propensos a lesionarse, cuando experimentaban 
aumentos significativos de problemas diarios, durante la semana de la lesión y la semana anterior.

El estudio de los sucesos vitales estresantes ha proporcionado una mayor verificación a la relación entre el estrés y la lesión. Existe una importante línea de investigación en este sentido, donde los resultados encontrados, a excepción del estudio de Williams, Haggert, Tonymon y Wadsworth (1986), indican que los deportistas con niveles altos de estrés son más propensos a lesionarse que aquellos deportistas con niveles bajos (Bramwell, Masuda, Wagner \& Holmes, 1975; Coddington \& Troxel, 1980; Cryan \& Alles, 1983; Díaz et al., 2004; Lysens, Auweele \& Ostyn, 1986; May, Veach, Reed \& Griffey, 1985; Passer \& Seese, 1983). La mayoría de autores que han estudiado la relación entre estrés psicosocial y vulnerabilidad a la lesión deportiva, han estudiado los sucesos vitales estresantes, diferenciando entre sucesos vitales negativos (SVN), sucesos vitales positivos (SVP) y sucesos vitales totales, como suma de los dos anteriores (SVT).

El estudio de los SVN ha permitido establecer que, en general, los deportistas que han experimentado este tipo de sucesos, tienen una mayor probabilidad de lesionarse (Hardy \& Riehl, 1988; Meyer, 1995; Palmeira, 1998; Passer \& Seese, 1983; Petrie, 1993a; Smith, Smoll \& Ptacek, 1990; Smith, Ptacek \& Smoll, 1992). Además, algunos trabajos (Hardy \& Riehl, 1988) han encontrado que las diferencias de género y la especificidad del deporte practicado influían en la relación entre lesión y estrés, aunque las diferencias de género no han aparecido en otros estudios (Ford, Eklund \& Gordon, 2000).

El estudio de los SVP proporciona algunos resultados paradójicos. Mientras algunas investigaciones encuentran una relación positiva entre SVP y tasa de lesiones (Blackwell \& McCullagh, 1990; Hanson et al., 1992; Petrie, 1993b), otras indican que el hecho de haber experimentado SVP podría servir de protector de la salud, y por lo tanto minimizar el riesgo de lesión (Palmeira, 1998; Zach, 1997).

Algunos estudios no han tenido en cuenta la diferenciación entre SVN y SVP, o bien han relacionado el total de los sucesos experimentados desde una perspectiva cuantitativa. En general, indican que determinados sucesos vitales tienen una gran carga de estrés para los deportistas (Coddington \& Troxell, 1980; Cryan \& Alles, 1983; Hardy, Prentice, Kirsanoff, Richman \& Rosenfeld, 1987; Kerr \& Minden, 1988; Passer \& Seese, 1983; Smith, Smoll \& Ptacek, 1990), y que la diferenciación entre sucesos estresantes vitales generales (muerte de miembros de la familia, cambios de residencia) y sucesos específicos del contexto deportivo (problemas con entrenadores, suplencia, etc.), podría ser importante para determinar la influencia de unos y otros en las lesiones (Bramwell et al., 1975; Kerr \& Minden, 1988).

En general, la revisión de la literatura científica acerca de la relación entre estrés psicosocial y lesión deportiva, indica que los deportistas con niveles altos de estrés tienden a sufrir lesión entre dos y cinco veces más, que los deportistas con niveles bajos de estrés, y que el riesgo de lesión aumenta en proporción directa al nivel de estrés experimentado (Andersen \& Williams, 1997; Byrd, 1993 como se cita en Williams \& Andersen, 1998; Fawkner, 1995; Kolt \& Kirkby, 1996; Meyer, 1995; Perna \& McDowell, 1993; Petrie, 1993a, 1993b; Thompson \& Morris, 1994; Williams \& Roepke, 1993). Aunque en algunos trabajos no se han encontrado relaciones entre los sucesos vitales y la lesión (Aygul, Akova \& Gur, 2001; Hardy, Richman \& Rosenfeld, 1991; Laws \& Donna, 2001; Lysens et al., 1986; Petrie \& Stoever, 1995; Williams, Tonymon \& Wadsworth, 1986), o bien solo las han encontrado al considerar el nivel de gravedad de las lesiones (Blackwell \& McCullagh, 1990; Hanson et al., 1992; Hardy, O’Connor \& Geisler, 1990; Hardy \& Riehl, 1988; Kerr \& Minden, 1988; Meyer, 1995; Petrie, 1992, 1993a). Considerando que las diferencias en algunos aspectos conceptuales, como los criterios de lesión, el nivel competitivo, los instrumentos de evaluación del estrés, o los diseños llevados a cabo (Williams \& Roepke, 1993), han proporcionado resultados excesivamente dispares, y a veces contradictorios (Junge, 2000; Olmedilla, Ortega, Abenza \& Boladeras, 2011), se han seguido algunas de las recomendaciones metodológicas de algunos autores 
(Díaz et al., 2004; Petrie \& Falkstein, 1998), como la evaluación en un mismo periodo temporal de sucesos vitales experimentados y lesiones sufridas, la especificidad del deporte y la homogeneidad de la muestra respecto al género. Así, la presente investigación se planteó como objetivos:

- Relacionar el estrés percibido por los tenistas y la frecuencia, tipo y gravedad de lesiones sufridas.

- Determinar si un nivel alto de estrés percibido se relaciona con un mayor número de lesiones, o con lesiones de mayor gravedad.

- Determinar si la evaluación que hace el tenista (positiva o negativa) de los sucesos vitales se relaciona con la frecuencia, tipo y gravedad de lesiones sufridas.

\section{Método}

\section{Tipo de investigación y diseño}

La presente es una investigación de tipo no experimental-descriptivo-correlacional, con un diseño de corte transversal, en el cual todas las variables en estudio fueron evaluadas en un único momento, y de manera retrospectiva, con el fin de descubrir las relaciones entre ellas (Hernández, Fernández \& Baptista, 2003)

\section{Participantes}

Participaron en el estudio un total de 63 jugadores de tenis, con edades comprendidas entre los 16 y los 49 años $(M=31.62, \mathrm{DT}=8.93)$. Todos ellos pertenecían a clubes de tenis de la Comarca Bajo Vinalopó de la provincia de Alicante (España), inscritos en campeonatos intraclub e interclubes. La mayoría de tenistas tenían una gran experiencia en la práctica del tenis; así, 30.16 \% lo practicaba desde hacía más de 12 años, $25.40 \%$ entre 8 y 12 años, $28.57 \%$ entre 4 y 8 años, $12.70 \%$ entre 1 y 4 años, y solo $3.17 \%$ tenía una experiencia de menos de 1 año. Respecto al tiempo de práctica del tenis, la mayoría (52.38 \%) lo practicaba entre 1 y 3 horas semanales, $22.22 \%$ lo practicaba menos de una hora, $14.29 \%$ lo practicaba entre 3 y 5 horas, $7.94 \%$ lo practicaba entre 5 a 8 horas, y solo 3.17 \% lo hacía durante más de 8 horas semanales.

\section{Instrumentos}

Para la evaluación del estrés psicosocial se utilizó el Cuestionario de Sucesos Vitales (CSV) de Sandín y Chorot (1987). Este cuestionario consta de una lista de 65 sucesos referidos a 11 áreas diferentes (trabajo, salud, amor, matrimonio/pareja, familia, hijos, social, legal, finanzas, residencia, académico). El CSV utiliza un sistema de valoración dimensional (no escalar), en línea por lo sugerido por Richards S. Lazarus (Sandín, 1999), que permite evaluar el número de sucesos experimentados durante un periodo de tiempo determinado, el nivel de estrés o tensión experimentada y la valencia de los sucesos (positivo o negativo), mediante la valoración que el sujeto hace de los diferentes sucesos y la interpretación positiva o negativa de los mismos. Su confiabilidad ha sido estudiada en muestras españolas, oscilando el alfa de Cronbach entre 0.49 y 0.62 para las puntuaciones totales del cuestionario, y se han utilizado la sintomatología somática, la enfermedad física y los trastornos de ansiedad como criterios de su validez predictiva (Sandín \& Chorot, 1993).

Para la evaluación de las lesiones de los tenistas, se utilizó un cuestionario de autoinforme, partiendo del utilizado en otros estudios (Abenza, Olmedilla \& Ortega, 2010; Díaz et al., 2004; Olmedilla, Ortega, Prieto \& Blas, 2009) en el que se registraban el número, tipo y gravedad de lesiones sufridas durante la temporada deportiva anterior (aproximadamente un año), además de la situación de lesionado o no lesionado en el momento del estudio. Para la valoración de la gravedad de las lesiones se siguió un criterio funcional (Díaz et al., 2004; Olmedilla, García-Montalvo \& MartínezSánchez, 2006), diferenciando entre lesiones leves (al menos interrumpen un día de entrenamiento y requieren tratamiento), lesiones moderadas (obliga al tenista a interrumpir durante al menos una semana sus entrenamientos y competiciones, y 
requieren tratamiento), lesiones graves (suponen uno o dos meses de baja deportiva, a veces hospitalización, e incluso intervención quirúrgica) y lesiones muy graves (producen una disminución del rendimiento del tenista de manera permanente, precisando rehabilitación constante para evitar empeoramiento).

\section{Procedimiento}

Para la realización del presente trabajo se contactó con los responsables y directivos de cada club de tenis. Se estableció una entrevista con ellos, en la que se explicó el objetivo del estudio, se propuso el método de obtención de datos y se solicitó permiso para llevarla a cabo. Antes de iniciar el proceso de administración de los cuestionarios, se solicitó permiso a los propios tenistas, informándoles de la confidencialidad y del anonimato de los datos, y se firmó un consentimiento informado. Los cuestionarios se administraron en las pistas de entrenamiento de forma individual o colectiva, en cada uno de los clubes deportivos.

\section{Análisis estadístico}

En este estudio se ha utilizado una metodología correlacional. Los datos descriptivos se han realizado mediante medias y desviaciones típicas. Para el análisis correlacional se ha utilizado el coeficiente de correlación de Pearson y técnicas estadísticas de contrastes $t$ de Student para medias procedentes de muestras independientes. El tratamiento de los datos se llevó a cabo mediante el paquete estadístico SPSS 15.0 para Windows.

\section{Resultados}

En la Tabla 1 se puede observar la distribución de la muestra en relación con las lesiones, concretamente, la situación actual del tenista respecto a si está o no lesionado y la gravedad de lesión que sufre, y la historia de lesiones sufridas durante la práctica del tenis. Casi la mitad de los deportistas de la muestra no se encuentran lesionados en el momento del estudio, destacando el hecho de que más de un $20 \%$ sufre una lesión moderada o grave. Respecto a las lesiones sufridas con anterioridad, casi un tercio (30.2\%) no se ha lesionado nunca.

En la Tabla 2 se indican en frecuencia y porcentajes la zona lesionada de los tenistas y el tipo de lesiones sufridas por los tenistas. Como cabría esperar, la mayoría de lesiones se producen en diferentes zonas de los miembros superiores (66.67\%); además, es de destacar igualmente el porcentaje de lesiones en piernas y tobillos (19.44\%). Por otro lado, destaca el hecho de que la gran mayoría de lesiones son musculares y tendinitis (un $81.94 \%$ ), solo una fractura (1.39\%), y ninguna contusión.

$\mathrm{Al}$ analizar la relación entre los sucesos vitales y las lesiones, solo se han encontrado relaciones significativas entre el grado de tensión psicológica experimentada y la evaluación de esta tensión, y las lesiones producidas en las muñecas $(r=0.315$;

TABLA 1

Situación actual del tenista respecto a estar o no lesionado y número de lesiones sufridas a lo largo de su práctica de tenis

\begin{tabular}{|c|c|c|c|c|c|}
\hline Lesión actual & Frecuencia & $\%$ & № de lesiones sufridas & Frecuencia & $\%$ \\
\hline No lesionado & 31 & 49.2 & Ninguna & 19 & 30.2 \\
\hline Lesión leve & 19 & 30.2 & Una & 25 & 39.7 \\
\hline Lesión moderada & 11 & 17.5 & Dos & 10 & 15.9 \\
\hline Lesión grave & 2 & 3.2 & Tres & 8 & 12.7 \\
\hline \multirow{2}{*}{ Total } & \multirow{2}{*}{63} & \multirow{2}{*}{100} & Cuatro & 1 & 1.6 \\
\hline & & & Total & 63 & 100 \\
\hline
\end{tabular}

Fuente: elaboración propia. 


\section{TABLA 2}

Frecuencia y porcentaje de la zona lesionada y del tipo de lesiones sufridas por los tenistas

\begin{tabular}{lcclcc}
\hline \multicolumn{1}{c}{ Zona lesionada } & Frecuencia & Porcentaje & Tipo de lesión & Frecuencia & Porcentaje \\
\hline Hombros & 24 & 33.33 & Musculares & 33 & 45.83 \\
Brazos & 10 & 13.89 & Tendinitis & 26 & 36.11 \\
Codos & 11 & 15.28 & Esguinces & 8 & 11.11 \\
Muñecas & 3 & 4.17 & Fracturas & 1 & 1.39 \\
Caderas & 1 & 1.39 & Contusiones & 0 & 0 \\
Lumbares & 4 & 5.56 & Otras & 4 & 5.56 \\
Piernas & 8 & 11.11 & & & \\
Rodillas & 5 & 6.94 & & & \\
Tobillos & 6 & 8.33 & & 72 & 100.00 \\
Total & 72 & 100.00 & Total & & \\
\hline
\end{tabular}

Fuente: elaboración propia.

$a \leq 0.05 ; y(r=0.251 ; a \leq 0.05$, respectivamen-

te). Y una relación marginalmente significativa con las lesiones ocasionadas en los tobillos $(r=0.246$; $a=0.052$ ) (Tabla 3).

TABLA 3

Correlaciones entre las dimensiones del CSV y las lesiones

\begin{tabular}{|c|c|c|c|}
\hline & & $\begin{array}{l}\text { Evaluación de la tensión } \\
\text { (Sumatorio de CSV2) }\end{array}$ & $\begin{array}{l}\text { Tensión psicológica } \\
\text { (Sumatorio de CSV1) }\end{array}$ \\
\hline \multirow{3}{*}{$\begin{array}{l}\text { A menor puntuación la evalua- } \\
\text { ción de la tensión será más posi- } \\
\text { tiva (Sumatorio de CSV2) }\end{array}$} & Cor. Pearson & 1 & $0.968(* *)$ \\
\hline & Sig. (bilateral) & & 0.000 \\
\hline & $N$ & 63 & 63 \\
\hline \multirow{3}{*}{$\begin{array}{l}\text { A mayor puntuación mayor ten- } \\
\text { sión (Sumatorio de CSV1) }\end{array}$} & Cor. Pearson & $0.968(* *)$ & 1 \\
\hline & Sig. (bilateral) & 0.000 & \\
\hline & $\mathrm{N}$ & 63 & 63 \\
\hline \multirow{3}{*}{$\begin{array}{l}\text { Número de lesiones ocasionadas } \\
\text { en las muñecas }\end{array}$} & Cor. Pearson & $0.315(*)$ & $0.251(*)$ \\
\hline & Sig. (bilateral) & 0.012 & 0.047 \\
\hline & $N$ & 63 & 63 \\
\hline \multirow{3}{*}{$\begin{array}{l}\text { Número de lesiones ocasionadas } \\
\text { en los tobillos }\end{array}$} & Cor. Pearson & 0.246 & 0.183 \\
\hline & Sig. (bilateral) & 0.052 & 0.152 \\
\hline & $N$ & 63 & 63 \\
\hline
\end{tabular}

** La correlación es significativa al nivel 0,01 (bilateral).

* La correlación es significante al nivel 0,05 (bilateral).

a No se puede calcular porque al menos una variable es constante.

Fuente: elaboración propia. 
Debido a que las correlaciones entre las variables no han sido elevadas, y a la distribución asimétrica de la muestra, se ha optado por determinar un criterio de selección de un grupo de esta, donde aquellos tenistas del centil 75 (puntuaciones altas en el CSV), se han comparado con el grupo del centil 25 (puntuaciones bajas en el CSV), respecto a las lesiones sufridas. En la Tabla 4 se puede observar el análisis descriptivo previo de las dos dimensiones del CSV, la tensión psicológica y la evaluación de esta tensión.

Por ello, se seleccionan todos los casos que hayan obtenido una puntuación de 17 o mayor en la dimensión de tensión psicológica, y de 10 o mayor en la dimensión de evaluación de la tensión psicológica. Al relacionar el grupo de nivel alto de tensión psicológica con el grupo de nivel bajo, solo se aprecian relaciones significativas en el número de lesiones leves $(t(16)=2.264, p<0.05)$, que paradójicamente indican que los tenistas con mayor nivel de tensión psicológica, sufren menos lesiones leves que los de menor tensión psicológica (Tabla 5).

\section{TABLA 5}

Diferencias de medias y pruebas t entre los grupos de tensión normal y alta tensión psicológica y las lesiones

\begin{tabular}{lccccc}
\hline & $\begin{array}{c}\text { Tensión } \\
\text { Psicológica }\end{array}$ & $N$ & Media & $\begin{array}{c}\text { Desv. } \\
\text { típica }\end{array}$ & $\begin{array}{c}\text { Error } \\
\text { típ. de } \\
\text { la media }\end{array}$ \\
\hline $\begin{array}{l}\text { Número } \\
\text { de lesiones } \\
\text { leves }\end{array}$ & Baja & 6 & 0.66 & 0.81 & 0.333 \\
\hline
\end{tabular}

Fuente: elaboración propia.

Por último, al relacionar el grupo de nivel alto de evaluación negativa de los sucesos vitales, con el

TABLA 4. Análisis descriptivo de los valores de los tenistas de la muestra en las dimensiones del CSV

\begin{tabular}{lccc}
\hline & $\begin{array}{c}\text { A menor puntuación la evaluación de la tensión } \\
\text { será más positiva (Sumatorio de CSV2) }\end{array}$ & $\begin{array}{c}\text { A mayor puntuación mayor } \\
\text { tensión (Sumatorio de CSV1) }\end{array}$ \\
\hline$N \quad$ Válidos & 63 & 63 \\
& Perdidos & 1 & 1 \\
\hline Media & 8.15 & 14.12 \\
Mediana & 7.00 & 11.00 \\
Moda & 3.00 & 0.00 \\
Desv. típ. & 7.75 & 14.54 \\
Varianza & 60.13 & 211.46 \\
Asimetría & 2.36 & 2.85 \\
Error típ. de asimetría & 0.302 & 0.302 \\
Curtosis & 6.93 & 10.18 \\
Error típ. de curtosis & 0.595 & 0.595 \\
Mínimo & 0.00 & 0.00 \\
Máximo & 39.00 & 79.00 \\
\hline & 3.00 & 6.00 \\
Percentiles & 50 & 7.00 & 11.00 \\
& 75 & 10.00 & 17.00 \\
\hline
\end{tabular}

Fuente: elaboración propia. 
grupo de nivel bajo, se aprecia que el grupo de nivel alto ha tenido una media de lesiones más elevada en el número de esquinces $(t(61)=-2.570, p<$ $0.05)$ y en el número de lesiones ocasionadas en los tobillos $(t(61)=-2.442, p<0.05)($ Tabla 6$)$. TABLA 6

Diferencias de medias y pruebas t entre los grupos de tensión normal y alta tensión psicológica y las lesiones

\begin{tabular}{|c|c|c|c|c|c|}
\hline & $\begin{array}{c}\text { Evaluación } \\
\text { Negativa }\end{array}$ & $N$ & Media & $\begin{array}{l}\text { Desv. } \\
\text { típica }\end{array}$ & $\begin{array}{l}\text { Error } \\
\text { típ. de } \\
\text { la media }\end{array}$ \\
\hline \multirow{2}{*}{$\begin{array}{l}\text { Número de } \\
\text { esguinces }\end{array}$} & Baja & 45 & 0.04 & 0.20 & 0.03 \\
\hline & Alta & 18 & 0.33 & 0.68 & 0.16 \\
\hline \multirow{2}{*}{$\begin{array}{l}\text { Número de } \\
\text { lesiones en } \\
\text { los tobillos }\end{array}$} & Baja & 45 & 0.02 & 0.14 & 0.02 \\
\hline & Alta & 18 & 0.27 & 0.66 & 0.15 \\
\hline
\end{tabular}

Fuente: elaboración propia.

\section{Discusión}

En este trabajo el objetivo ha sido analizar la relación entre el estrés social percibido por los tenistas y las lesiones sufridas por estos. Para ello, se ha evaluado retrospectivamente, tanto el estrés social como las lesiones sufridas, durante el último año de práctica deportiva. Concretamente, se ha pretendido determinar si un nivel alto de estrés percibido, se relaciona con un mayor número de lesiones, y si esto es así, de qué tipo o nivel de gravedad eran; además, se ha pretendido determinar si la evaluación (positiva o negativa) que hacen los tenistas de los sucesos vitales, se relaciona con las lesiones sufridas.

Los resultados del estudio indican que al analizar la relación entre los sucesos vitales y las lesiones, se han encontrado relaciones entre el grado de tensión psicológica experimentada y la evaluación negativa de esta tensión, con las lesiones producidas en las muñecas. Además se puede apreciar una tendencia a la relación entre el grado de tensión psicológica experimentada y la evaluación negativa de esta tensión, con las lesiones ocasionadas en los tobillos. Estos resultados se encuentran en la línea de los hallados en las investigaciones que relacionan los SVN con las lesiones y que, en general, establecen que los deportistas que han experimentado este tipo de sucesos, tienen una mayor probabilidad de lesionarse (Hardy \& Riehl, 1988; Meyer, 1995; Palmeira, 1998; Passer \& Seese, 1983; Petrie, 1993a; Smith et al., 1990; Smith et al., 1992); además, es de destacar que, en este estudio, las relaciones se producen con las lesiones en muñecas, lo que parece ratificar la importancia de la especificidad del deporte, tal y como indican Hardy y Riehl (1988).

Por otro lado, cuando se han relacionado exclusivamente los sucesos vitales, sin tener en cuenta la valoración (positiva o negativa) de los tenistas, comparando el grupo de nivel alto de tensión psicológica con el grupo de nivel bajo, se han encontrado relaciones con el número de lesiones leves, aunque en este caso, y paradójicamente, los resultados indican que los tenistas con mayor nivel de tensión psicológica sufren menos lesiones (de gravedad leve) que los de menor tensión psicológica, en línea con algunos de los resultados paradójicos analizados al estudiar los sucesos vitales; así, la relación positiva entre SVP y tasa de lesiones encontrada por algunos autores (Blackwell \& McCullagh, 1990; Hanson et al., 1992; Petrie, 1993b), contrasta con la relación negativa encontrada por otros (Palmeira, 1998), hablando incluso de un factor de protección de salud (Zach, 1997). Este hecho podría sugerir que quizás un determinado nivel de tensión psicológica hasta sea bueno para la práctica competitiva, aunque la tensión evaluada con el CSV no es específica del deporte, lo que no ayuda mucho a comprender mejor las relaciones encontradas. Como es lógico el nivel de tensión percibido por un sujeto afecta a cualquier área de su actividad, incluyendo en este caso la deportiva; además, un cierto nivel de tensión podría ayudar al deportista a mejorar su rendimiento y a centrarse en su tarea, lo que de alguna manera estaría ayudando a reducir el nivel de vulnerabilidad a la lesión; sin embargo, niveles bajos de tensión psicológica podrían provocar una reducción de la capacidad atencional del sujeto, que en la práctica deportiva estaría incrementando la probabilidad de sufrir lesión del deportista (Andersen \& Williams, 1997; Rogers \& Landers, 2002, 
2005; Williams, Tonymon \& Andersen, 1990, 1991). En este sentido, debería tenerse en cuenta que la tensión psicológica en sí misma, no presupone la valoración negativa de la misma, como bien determinan los dos componentes de la ansiedad, el somático (neutro) y el cognitivo (que otorga valoración positiva o negativa). Aunque, en la línea de lo sugerido por otros autores (Dunn, Smith \& Smoll, 2001), los sucesos estresantes deben diferenciarse entre los que se consideran sucesos vitales generales y los sucesos específicos del deporte, y se deben evaluar de manera independiente para poder determinar la fuerza predictiva de unos y otros respecto a las lesiones deportivas; en su estudio los autores encontraron que los sucesos vitales específicos del deporte, mostraban una relación más fuerte con las lesiones que los sucesos vitales generales.

Por último, al relacionar el grupo de nivel alto de evaluación negativa de los sucesos vitales con el grupo de nivel bajo, se aprecia que el grupo de nivel alto ha tenido una media de lesiones más elevada en el número de esquinces y en el número de lesiones ocasionadas en los tobillos, en la línea de lo encontrado en trabajos anteriores (Palmeira, 1998; Petrie, 1993a; Smith et al., 1990; Smith et al., 1992). En general, durante la última década los trabajos de investigación de los SVN han incorporado diseños multivariantes, que permiten conocer mejor las relaciones entre estos y otros factores psicológicos que están afectando el nivel de vulnerabilidad del deportista a la lesión. Quizás, la línea de trabajos más representativa sea la que relaciona los SVN con los recursos de afrontamiento y su incidencia en el estrechamiento periférico producido por el estrés; así, los recursos de afrontamiento y un apoyo social positivo de los deportistas suelen ayudar a mitigar los efectos del estrés psicosocial (Smith, 2001; Williams \& Andersen, 1997), aunque en algunos trabajos no se ha encontrado esta relación (Aygul et al., 2001). Uno de los aspectos psicológicos que se han incorporado recientemente al estudio de las relaciones entre estrés y lesiones, ha sido el estado de ánimo, que además parece ser una línea muy interesante de trabajo. Así, se ha estudiado el estado de ánimo como predictor de lesiones (Galambos, Terry, Moyle \& Locke, 2005; Kleinert, 2007), y se han estudiado los SVN como predictores de perturbaciones del estado de ánimo de los deportistas lesionados durante su recuperación (Albinson \& Petrie, 2003).

Parece que la mayoría de autores ya consideran que la lesión deportiva es causada por diferentes factores (Knowles, Marshall \& Guskievicz, 2006), y que en esta multicausalidad el factor psicológico tiene su relevancia, donde el estrés parece jugar un papel destacado (Buceta, 2008). Además, la publicación de trabajos en los que se determina la influencia de los programas de intervención psicológica en la reducción de lesiones deportivas (Johnson, Ekengren \& Andersen, 2005; Maddison \& Prapavessis, 2005; Noh, Morris \& Andersen, 2007), resulta un acicate a la consideración de la importancia del control del estrés en la prevención.

\section{Limitaciones y sugerencias de futuro}

A pesar de haber aportado algunos avances a planteamientos anteriores, como el estudio de los sucesos vitales durante el mismo periodo temporal (último año de práctica deportiva), la homogeneización de la especificidad del deporte (tenis), o la unificación respecto al género (masculino), y de cubrir un vacío respecto a este tipo de estudios en el ámbito latinoamericano (solo se conocen los trabajos de Palmeira en 1998 y de Díaz et al. en el 2004)), es necesario considerar que investigaciones futuras deberían incrementar el número de participantes, aún conociendo la dificultad de todo trabajo realizado "a pie de pista". Ya que el tipo de muestreo utilizado en esta investigación no permite la generalización de los resultados, solo es una referencia que puede guiar futuras investigaciones.

Por otro lado, quizá sea interesante el estudio del estrés cotidiano, de los llamados "problemas diarios"; en este sentido, y tal y como indican Williams y Andersen (1998), los descubrimientos de Byrd (1993) y Fawkner (1995) sugieren evaluar los cambios de los problemas en una secuencia semanal y anotar sus incrementos, lo que puede 
ofrecer una medida más relevante del estrés diario y el riesgo de sufrir lesión.

Sería muy importante considerar los sucesos vitales de manera separada; por un lado, aquellos que hacen referencia a aspectos generales de la vida del deportista y, por otro, los sucesos específicos del ámbito deportivo; además, incorporar con cautela la valoración subjetiva del impacto del suceso, ya que los datos aportados por algunos trabajos parecen indicar que los sucesos vitales, sean positivos o negativos, pueden ser estresantes y perjudiciales. En este sentido, Petrie (1993b) sugirió que los sucesos vitales que los deportistas valoraban inicialmente como positivos (un gran cambio en el nivel de responsabilidad en el equipo, recibir una beca deportiva, etc.), podían producir en el futuro un mayor estrés, debido a la presión añadida por las expectativas de un mejor rendimiento o aportación al equipo, produciendo una valoración cognitiva negativa de las situaciones deportivas y, por tanto, un mayor riesgo de lesión.

Igualmente, es necesario plantear diseños de investigación multivariantes, incorporando el estudio de variables de personalidad, por ejemplo, y otros aspectos psicológicos, en la línea de las últimas investigaciones realizadas en el ámbito anglosajón y de lo sugerido en el modelo global psicológico de las lesiones deportivas de Olmedilla y García-Mas (2009); así como analizar las estrategias de afrontamiento que utilizan los deportistas y que se relacionan con la prevención o recuperación de lesiones (Abenza, Olmedilla, Ortega \& Esparza, 2011; Ali, Marivain, Héas \& Boulvais, 2006). Es de destacar, que el trabajo de Palmeira (1998) relaciona algunos aspectos de la personalidad (introversión, extraversión) con los sucesos vitales, encontrando que, tanto en los jugadores profesionales de baloncesto como en las gimnastas (gimnasia rítmica), la extroversión reducía la influencia de los SVN y que este se asociaba a mayores tasas de lesión.

Por último, la realización de estudios específicos en nuestro ámbito posibilitará una mejor comprensión del estrés y la relación entre los distintos aspectos de este y las lesiones deportivas, lo que permitirá diseñar programas de intervención psicológica más ajustados para controlar el estrés y minimizar los riesgos de lesión, tal y como en otros contextos han implementado algunos autores (Davis, 19991; Johnson et al., 2005; Maddison \& Prapavessis, 2005; Noh et al., 2007).

\section{Referencias}

Abenza, L., Olmedilla, A. \& Ortega, E. (2010). Efectos de las lesiones sobre las variables psicológicas en futbolistas juveniles. Revista Latinoamericana de Psicología, 42 (2), 225-236.

Abenza, L., Olmedilla, A., Ortega, E. \& Esparza, F. (2011). Construcción de un registro de conductas de adherencia a la rehabilitación de lesiones deportivas. Revista de Psicología del Deporte, 20 (2), 455-476.

Albinson, C. B. \& Petrie, T. A. (2003). Cognitive appraisals, stress, and coping: Preinjury and postinjury factors influencing psychological adjustment to sport injury. Journal of Sport Rehabilitation, 12, 306-322.

Ali, M., Marivain, T., Hèas, A. \& Boulvais, H. (2006). Analysis of coping strategies used by players of tennis men and women toward a severe athletic injury. Annales Médico-Psychologiques, 10, 1016.

Andersen, M. B. \& Williams, J. M. (1988). A model of stress and athletic injury: Prediction and prevention. Journal of Sport and Exercise Psychology, 10, 294-306.

Andersen, M. B. \& Williams, J. M. (1997). Athletic injury, psychosocial factors, and perceptual changes during stress. Journal of Sports Sciences, 17 (9), 735-741.

Aygul, Z. S, Akova, B. \& Gur, H. (2001). The relationship of stress and stress management factors with injury in soccer players. Turkish Journal of Sports Medicine, 36 (2), 71-80.

Blackwell, B. \& McCullagh, P. (1990). The relationship of athletic injury to life stress, competitive anxiety and coping resources. Athletic Training, 25, 23-27.

Bramwell, S. T., Masuda, M., Wagner, N. N. \& Holmes, T. H. (1975). Psychological factors in athletic injuries. Journal of Human Stress, 2, 6-20.

Buceta, J. M. (2008, diciembre). Aproximación psicológica a la lesión deportiva. I Simposium Nacional 
de Psicología del Deporte, Lesiones deportivas, prevención y rehabilitación, Universidad Católica San Antonio de Murcia, España.

Coddington, R. D. \& Troxel, J. R. (1980). The effects of emotional factors on football injury rates: A pilot study. Journal of Human Stress, 7, 3-5.

Cryan, P. D. \& Alles, W. F. (1983). The relationship between stress and college football injuries. Journal of Sports Medicine, 23, 52-58.

Chiriboga, D. A. (1989). The measurement of stress exposure in later life. In K. S. Markides \& C. L. Cooper (Eds.), Aging, stress and health (pp. 13-41). Chichester: John Wiley \& Sons.

Davis, J. O. (1991). Sport injuries and stress management: An opportunity for research. The Sport Psychologist, 5, 175-182.

Díaz, P., Buceta, J. M. \& Bueno, A. M. (2004). Situaciones estresantes y vulnerabilidad a las lesiones deportivas: un estudio con deportistas de equipo. Revista de Psicología del Deporte, 14, 7-24.

Dunn, E. C., Smith, R. E. \& Smoll, F. L. (2001). Do sport-specific stressors predict athletic injury? Journal of Science and Medicine in Sport, 4 (3), 283-291.

Fawkner, H. J. (1995). Predisposition to injuries in athletes: The role of psychosocial factors. Unpublished master's thesis, University of Melbourne, Australia.

Feldman, L., Gonçalves, L., Chacón-Puignau, G., Zaragoza, J., Bagés, N. \& De Pablo, J. (2008). Relaciones entre estrés académico, apoyo social, salud mental y rendimiento académico en estudiantes universitarios venezolanos. Universitas Psychologica, 7 (3), 739-751.

Fernández, L., Siegrist, J., Rödel, A. \& Hernández, R. (2003). El estrés laboral un nuevo factor de riesgo ¿qué sabemos y qué podemos hacer? Atención Primaria, 31 (18), 524-26.

Ford, I. W., Eklund, R. C. \& Gordon, S. (2000). An examination of psychosocial variables moderating the relationship between life stress and injury time-loss among athletes of a high standard. Journal of Sports Sciences, 18 (5), 301-312.

Galambos, S. A., Terry, P. C., Moyle, G. M. \& Locke, S. A. (2005). Psychological predictors of injury among elite athletes. British Journal of Sports Medicine, 39 (6), 351-354.
Hanson, S. J., McCullagh, P. \& Tonymon, P. (1992). The relationship of personality characteristics, life stress, and coping resources to athletic injury. Journal of Sport and Exercise Psychology, 14, 262-272.

Hardy, C. J. \& Riehl, M. A. (1988). An examination of the life stress-injury relationship among noncontact sport participants. Behavioral Medicine, 14, 113-118.

Hardy, C. J., O Connor, K. A. \& Geisler, P. R. (1990). The role of gender and social support in the life stress injury relationship [Abstract]. Proceedings of the $5^{\text {th }}$ annual Conference of the Association for the Advancement of Applied Sport Psychology, 51.

Hardy, C. J., Prentice, W. E., Kirsanoff, M. T., Richman, J. M. \& Rosenfeld, L. B. (1987, June). Life stress, social support and athletic injury: In search of relationships. In J. M. Williams (Chair), Psychological factors in injury occurrence. Symposium conducted at the meeting of the NASPSPA, Vancouver, British Columbia, Canada.

Hardy, C. J., Richman, J. M. \& Rosenfeld, L. B. (1991). The role of social support in the life stress/injury relationship. The Sport Psychologist, 5, 128-139.

Hernández, M., Fernández, C. \& Baptista, P. (2003). Metodología de la investigación (3a. ed.). México: McGraw-Hill.

Holmes, T. H. (1970, February). Psychological screening in football injuries. Papers presented at workshop (pp. 211-214). (Sponsored by Subcommittee on Athletic Injuries, Committee on the Skeletal System, Division of Medical Sciences, National Research Council, Feb. 1969). Washington, DC: National Academy of Sciences.

Holmes, T. H. \& Rahe, R. H. (1967). The Social Readjustment Rating Scale. Journal of Psychosomatic Research, 11, 213-218.

Johnson, U., Ekengren, J. \& Andersen, M. B. (2005). Injury prevention in Sweden: Helping soccer player at risk. Journal of Sport $\mathcal{E}$ Exercise Psychology, 27, 32-38.

Junge, A. (2000). The influence of psychological factors on sports injuries: Review of the literature. American Journal of Sports Medicine, 28, 10-15.

Kaplan, H. B. (1996). Perspectives on psychosocial stress. In H. B. Kaplan (Ed.), Psychosocial stress (pp. 3-24). San Diego, CA: Academic Press. 
Kerr, G. \& Minden, H. (1988). Psychological factors related to the occurrence of athletic injuries. Journal of Sport and Exercise Psychology, 10, 167-173.

Kleinert, J. (2007). Mood states and perceived physical states as short term predictors of sport injuries: Two prospective studies. International Journal of Sport and Exercise Psychology, 5 (4), 340-351.

Knowles, S. B., Marshall, S.W. \& Guskiewicz, K. M. (2006). Issues in estimating risks and rates in sports injury research. Journal of Athletic Training, 41 (2), 207-215.

Kolt, G. \& Kirkby, R. (1996). Injury in Australian female competitive gymnasts: A psychological perspective. Australian Physiotherapy, 42, 121-126.

Laws, G. \& Donna, Y. (2001). The relationship of life stress, social support systems, and coping behaviors associated with athletic injury and performance in women's intercollegiate softball teams. Dissertation Abstracts International, 62 (2B), 1133.

Lazarus, R. \& Folkman, S. (1986). Estrés y procesos cognitivos. Madrid: Martínez Roca.

Lysens, R., Auweele, Y. V. \& Ostyn, M. (1986). The relationship between psychosocial factors and sports injuries. Journal of Sports Medicine and Physical Fitness, 26, 77-84.

Maddison, R. \& Prapavessis, H. (2005). A psychological approach to the prediction and prevention of athletic injury. Journal of Sport $\mathbb{E}$ Exercise Psycho$\log y, 27,289-310$.

Malhotra, S. K. \& Mehta, V. (2008). Role of stressful life events in induction or exacerbation of psoriasis and chronic urticaria. Indian Journal of Dermatology, Venereology and Leprology, 74, 594-599.

May, J. R., Veach, T. L., Reed, M. W. \& Griffey, M. S. (1985). A psychological study of health, injury and performance in athletes on the U.S. alpine ski team. Physician and Sports Medicine, 13, 111-115.

Meyer, K. N. (1995). The influence of personality factors, life stress, and coping strategies on the incidence of injury in long-distance runners. Unpublished master's thesis, University of Colorado, Boulder, United States.

Miller, T. W. (1988). Advances in understanding the impact of stressful life events on health. Hospital and Community Psychiatry, 39, 615-622.
Noh, Y.-E., Morris, T. \& Andersen, M. B. (2007). Psychological intervention for reduction of injury in ballet dancers. Research in Sports Medicine, 15, 13-32.

Olmedilla, A. \& García-Mas, A. (2009). El modelo global psicológico de las lesiones deportivas. Acción Psicológica, 6 (2), 77-91.

Olmedilla, A., García-Montalvo, C. \& Martínez-Sánchez, F. (2006). Factores psicológicos y vulnerabilidad a las lesiones deportivas: un estudio en futbolistas. Revista de Psicología del Deporte, 15 (1), 7-19.

Olmedilla, A., Ortega, E., Abenza, L. \& Boladeras, A. (2011). Lesiones deportivas y psicología: una revisión (2000-2009). Cuadernos de Psicología del Deporte, 11 (1), 45-57.

Olmedilla, A., Ortega, E., Prieto, J. M. \& Blas, A. (2009). Percepción de los tenistas respecto a los factores que pueden provocar lesiones: diferencias entre federados y no federados. Cuadernos de Psicología del Deporte, 9 (2), 7-18.

Olmedilla, A., Ortín, F. J. \& De la Vega, R. (2006). Lesiones deportivas y Psicología: análisis, investigación y propuestas de intervención. En E. J. Garcés de los Fayos, A. Olmedilla \& P. Jara (Coords.), Psicología y deporte (pp. 497-523). Murcia: Diego Marín.

Ortín, F. J., Garcés de los Fayos, E. J. \& Olmedilla, A. (2010). Influencia de los factores psicológicos en las lesiones deportivas. Papeles del Psicólogo, 31 (3), 143-154.

Palmeira, A. (1998). Antecedentes psicológicos de la lesión deportiva. Revista de Psicología del Deporte, 8 (1), 117-132.

Passer, M. W. \& Seese, M. D. (1983). Life stress and athletic injury: Examination of positive versus negative events and three moderator variables. Journal on Human Stress, 10, 11-16.

Perna, F. \& McDowell, S. (1993, October). The association of stress and coping with illness and injury among elite athletes. Paper presented at the annual meeting of the Association for the Advancement of Applied Sport Psychology, Montreal, Quebec, Canada.

Petrie, T. A. (1992). Psychosocial antecedents of athletic injuries: The effects of life stress and social support 
on female collegiate gymnasts. Behavioral Medicine, 18, 127-138.

Petrie, T. A. (1993a). The moderating effects of social support and playing status on the life stress-injury relationship. Journal of Applied Sport Psychology, $5,1-16$.

Petrie, T. A. (1993b). Coping skills, competitive trait anxiety, and playing status: Moderating effects on the life stress-injury relationship. Journal of Sport and Exercise Psychology, 15, 261-274.

Petrie, T. A. \& Falkstein, D. L. (1998). Methodological, measurement and statistical issues in research on sport injury prediction. Journal of Applied Sport Psychology, 10, 26-45.

Petrie, T. A. \& Stoever, S. (1995). Psychosocial antecedents of athletic injury: A temporal analysis [Abstract]. Journal of Applied Sport Psychology, 7, S99.

Rogers, T. J. \& Landers, D. M. (2002). Effects of lifeevent stress and hardiness on peripheral narrowing prior to competition. Journal of Sport and Exercise Psychology, 24 (Suppl.), S105.

Rogers, T .J. \& Landers, D. M. (2005). Mediating effects of peripheral vision in the life event stress/athletic injury relationship. Journal of Sport and Exercise Psychology, 27, 271-288.

Sandín, B. (1999). El estrés psicosocial. Madrid: UNED.

Sandín, B. \& Chorot, P. (1987). Cuestionario de Sucesos Vitales (CSV). Madrid: UNED.

Sandín, B. \& Chorot, P. (1993). Stress and anxiety: Diagnostic validity of anxiety disorders according to life events stress, ways of doping and physical symptoms. Psiquis, 14, 48-54.

Sarason, I. G., Johnson, J. H. \& Siegel, J. M. (1978). Assessing the impact of life changes: Development of the Life Experiences Survey. Journal of Consulting and Clinical Psychology, 46, 932-946.

Savery, L. K. \& Wooden, M. (1994). The relative influence of life events and hassles on work-related injuries: Some Australian evidence. Human Relations, 47, 283-305.

Selzer, M. L. \& Vinokur, A. (1974). Life events, subjective stress and traffic accidents. American Journal of Psychiatry, 131, 903-906.

Smith, A. M. (2001). Elite collegiate female athletes: A comparison between injured and no injured upper and lower division student-athletes on life-stress, competitive trait anxiety, and coping skills. Dissertation Abstracts International, 61 (11A), 4288.

Smith, R., Ptacek, J. \& Smoll, F. (1992). Sensation seeking, stress and adolescent injures: A test of stress buffering, risk-taking and coping skills hypotheses. Journal of Personality and Social Psychology, 62, 1016-1024.

Smith, R. E., Smoll, F. \& Ptacek, J. L. (1990). Conjunctive moderator variable and resiliency research: Life stress, social support and coping resources, and adolescent sport injuries. Journal of Personality and Social Psychology, 58, 360-370.

Stuart, J. C. \& Brown, B. M. (1981). The relationship of stress and coping ability to incidence of diseases and accidents. Journal of Psychosomatic Research, 25, 255-260.

Theorell, T. (1992). Critical life changes: A review of research. Psychotherapy and Psychosomatics, 57, 108-117.

Thompson, N. J. \& Morris, R. D. (1994). Predicting injury risk in adolescent football players: The importance of psychological variables. Journal of Paediatric Psychology, 19, 415-429.

Udry, E. \& Andersen, M. B. (2002). Athletic injury and sport behavior. In T. Horn (Ed.), Advances in Sport Psychology (pp. 529-553). Champaign, Il: Human Kinetics.

Williams, J. M. \& Andersen, M. B. (1986). The relationship between psychological factors and injury occurrence. In J. Heil (Chair), Psychological aspects of sport injury. Symposium conducted at the meeting of the NASPSPA, Scottsdale, AZ, United States.

Williams, J.M. \& Andersen, M. B. (1997). Psychosocial influences on central and peripheral vision and reaction time during demanding tasks. Behavioral Medicine, 26, 160-167.

Williams, J. M. \& Andersen, M. B. (1998). Psychological antecedents of sport injury: Review and critique of the Stress and Injury Model. Journal of Applied Sport Psychology, 10, 5-25

Williams, J. M., Haggert, J., Tonymon, P. \& Wadsworth, W. A. (1986). Life stress and prediction of athletic injuries in volleyball, basketball and cross-country running. In L. E. Unestahl (Ed.), Sport psychology 
in theory and practice (pp. 51-77). Orebro, Sweden: Veje Publishers.

Williams, J. M. \& Roepke, N. (1993). Psychology of injury and injury rehabilitation. In R. Singer, M. Murphey \& L. Tennant (Eds.), Handbook of research on sport psychology (pp. 815-838). New York: MacMillan.

Williams, J. M. Tonymon, P. \& Wadsworth, A. (1986). Relationship of life stress injury in intercollegiate volleyball. Journal of Human Stress, 12, 38-43.

Williams, J. M., Tonymon, P. \& Andersen, M. B. (1990). Effects of life-event stress on anxiety and peripheral narrowing. Behavioral Medicine, 16, 174-181.

Williams, J. M., Tonymon, P. \& Andersen, M. B. (1991). The effects of stressors and coping resources on anxiety and peripheral narrowing. Journal of Applied Psychology, 3, 126-141.

Zach, S. S. (1997). Relationships among perceived social support, psychological coping resources, life events stressors, and injury frequency and severity in elite Israeli athletes. Dissertation Abstracts International, 58 (3A), 0799. 